\title{
The Weak Lensing Bispectrum Induced By Gravity
}

\author{
D. Munshi ${ }^{a}$, T. Namikawa ${ }^{b}$, T. D. Kitching ${ }^{a}$, J. D. McEwen ${ }^{a}$,

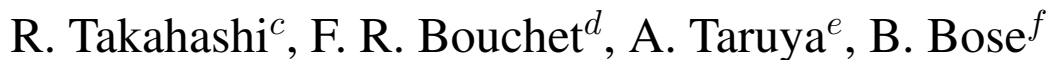 \\ ${ }^{a}$ Mullard Space Science Laboratory, University College London, Holmbury St Mary, Dorking, Surrey RH5 6NT, UK \\ ${ }^{b}$ Department of Applied Mathematics and Theoretical Physics, University of Cambridge, Wilberforce Road, Cambridge CB3 OWA, UK \\ e Faculty of Science and Technology, Hirosaki University, 3 Bunkyo-cho, Hirosaki, Aomori, 036-8561, Japan \\ ${ }^{d}$ Institut d'Astrophysique de Paris, UMR 7095, CNRS \& Sorbonne Universit, 98 bis Boulevard Arago, F-75014 Paris, France \\ e Center for Gravitational Physics, Yukawa Institute for Theoretical Physics, Kyoto University, Kyoto 606-8502, Japan \\ ${ }^{f}$ Departement de Physique, Universite de Geneve, 24 quai Ernest-Ansermet, CH-1211 Geneve 4, Switzerland
}

11 October 2019

\begin{abstract}
Recent studies have demonstrated that secondary non-Gaussianity induced by gravity will be detected with a high signal-to-noise $(\mathrm{S} / \mathrm{N})$ by future and even by on-going weak lensing surveys. One way to characterise such non-Gaussianity is through the detection of a non-zero three-point correlation function of the lensing convergence field, or of its harmonic transform, the bispectrum. A recent study analysed the properties of the squeezed configuration of the bispectrum, when two wavenumbers are much larger than the third one. We extend this work by estimating the amplitude of the (reduced) bispectrum in four generic configurations, i.e., squeezed, equilateral, isosceles and folded, and for four different source redshifts $z_{s}=0.5,1.0,1.5,2.0$, by using an ensemble of all-sky high-resolution simulations. We compare these results against theoretical predictions. We find that, while the theoretical expectations based on widely used fitting functions can predict the general trends of the reduced bispectra, a more accurate theoretical modelling will be required to analyse the next generation of all-sky weak lensing surveys. The disagreement is particularly pronounced in the squeezed limit.
\end{abstract}

Key words: : Cosmology- Weak lensing - Methods: analytical, statistical, numerical

\section{INTRODUCTION}

Thanks to recently completed Cosmic Microwave Background (CMB) experiments, such as the Planck space missior ${ }^{1}$ Planck Collaboration 2016a 2018), we now have a standard model of cosmology. There are however many outstanding questions that remain unanswered including, but not limited to, the nature of dark matter and dark energy; or possible modifications of General Relativity (GR) on cosmological scales (Joyce et al. 2012: Clifton et al. 2012, Planck Collaboration 2016b). In addition the sum of the neutrino masses (Lesgourgues et al. 2006) remains unknown. Not to mention aspects of the generation of the primordial fluctuations. Fortunately it is expected that the weak lensing surveys in operation, including Dark Energy Survey ${ }^{2}$ DES) (Abbott et al. 2015), Dark Energy Spectroscopic Instruments (DESI $3^{3}$ Subaru Hyper-Suprime Cam ${ }^{4}$ KiDS(Kujiken et al 2015) and near-future CFHTLS5 as well as Stage-IV large scale structure (LSS) sur-

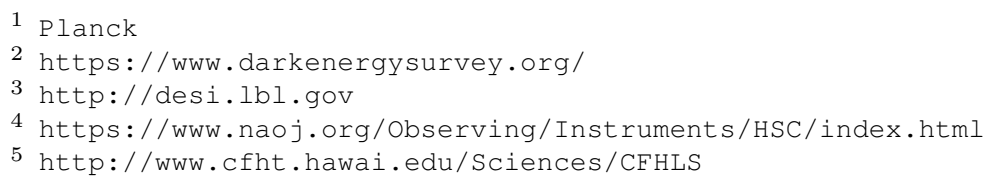

(c) 0000 RAS 


\section{Munshi et al.}

veys such as Euclid ${ }^{6}$ Laureijs et al 2015), the Large Synoptic Survey Telescope $\AA^{7} \mid$ Tyson et al 2015) and the Wide Field InfraRed Survey Telescope(WFIRST 2015) will provide some clues or even answers to many of the questions that cosmology is facing.

Weak lensing (the minute distortions in the images of the distant galaxies by the intervening large-scale structure) allows to extract information about the clustering of the intervening mass distribution in the Universe (Munshi et al. 2008). The weak lensing surveys are complementary to spectroscopic galaxy redshift surveys such as BOSS ${ }^{8}$ Eisenstein et al. 2011) or Wiggle $2^{9}$ Drinkwater et al. 2010) as they provide an unbiased picture of the underlying dark matter distribution, whereas the galaxies and other biased tracers require a difficult modelling of the light to mass relationship (Desjacques, Jeong \& Schmidt 2016) whose uncertainty is hard to assess precisely.

One challenge for weak lensing is that much of the information lies at small scales where clustering is non-linear and non-Gaussian (Bernardeau et al 2002), and theoretical predictions are challenging. Another challenge is that the statistical estimates of cosmological parameters based on power spectrum analysis are typically degenerate, e.g., $\sigma_{8}$ (the amplitude of mass density fluctuation) versus $\Omega_{M}$ (matter density parameter); to overcome these degeneracies, external data sets e.g., CMB, and the addition of tomographic or 3D (Castro, Heavens \& Kitching 2005) information is often used. In order to address both of these challenges an alternative procedure is to use higher-order statistics that probe the (quasi) non-linear regime (Munshi et al. 2011, Munshi, Valageas, Barber 2004, Munshi, Heavens, Coles 2010, Munshi et al. 2011, Valageas, Munshi, Barber 2005, Valageas, Barber, Munshi 2004, Byun et al. 2017; Song et al. 2015).

Previously it has been noted that even in the absence of any primordial non-Gaussianity (Bartolo et al. 2004), gravitational clustering induces mode couplings that results in a secondary non-Gaussianity which is more pronounced on smaller scales. Thus a considerable amount of effort has been invested in understanding the gravity induced secondary non-Gaussianity from weak lensing surveys. These statistics include the lower order cumulants (Munshi \& Jain|2001) and their correlators (Munshi|2000); the multispectra including the skew-spectrum (Munshi \& Heavens 2010) and kurtosis spectra (Munshi et al.2011) as well as the entire PDF (Munshi \& Jain 2000) and the statistics of hot and cold spots. Future surveys such as Euclid will be particularly interesting in this regard. With its large fraction of sky-coverage it will be able to detect the gravity induced non-Gaussianity with a very high signal-to-noise $(\mathrm{S} / \mathrm{N})$. It is also worth mentioning here that, in addition to breaking the degeneracy between cosmological parameters, higher-order statistics are also important for a better understanding of the covariance of lower-order estimators (Valageas, Barber, Munshi 2004). The construction of morphological estimators such as the Minkowski Functionals are discussed in (Munshi et al. 2012).

The large fraction of sky covered with Euclid will enable us to move from a real-sky correlation function analysis to a more convenient spectral analysis in the harmonic domain. The methods developed for analysing the CMB data will be helpful in this regard. Several such estimators were developed and were used to analyse the CMB maps generated by the Planck satellite (Komatsu, Spergel, Wandelt 2005 . Fergusson, Ligouri \& Shellard 2016, Munshi \& Heavens 2010, Bucher, Racined, van Tent 2016) and produced the currently best available constraints on non-Gaussianity in inflationary models as well as resulted in the detection of secondary non-Gaussianity (Planck Collaboration 2016a).

In a recent paper a new set of estimators that are particularly sensitive to the squeezed state of the bispectra also known as the Integrated Bispectra (IB) were proposed (Munshi et al.2019). These estimators are interesting because of their simplicity, and ease of implementation. Such estimators have also been used in 3D for quantifying galaxy clustering (Chiang et al. 2015) as well as in $1 \mathrm{D}$ to probe Lyman- $\alpha$ absorption features (Chiang \& Slosar 2018, Chinag et al. 2017). Here, we go beyond the squeezed configuration and extend the results to other shapes that include equilateral, folded and isosceles configurations. We use the techniques developed in (Namikawa et al. 2018) for CMB lensing. Using numerical ray-tracing simulations we check the validity of various approximations in the construction of such estimators.

This paper is organized as follows. In 12 we briefly summarize the theoretical results (for flat-sky as well as in all-sky limit). The simulations are presented in $\$ 3$ The results are presented in $\$ 4$ Finally, the discussions are presented in $\$ 5$

\section{WEAK LENSING CONVERGENCE, BISPECTRUM AND ESTIMATORS}

One of the first estimators developed for the study of non-Gaussianity is also know as the KSW estimator (Komatsu, Spergel, Wandelt 2005) which is a one-point estimator and is based on separable template fitting. It is computationally fast but being an one-point estimator it can not differentiate independent contributions to non-Gaussianity.

The skew-spectrum estimator developed in (Munshi \& Heavens 2010) provides a power spectrum related to the bispectrum. It is an extension of the one-point KSW estimator and retains more information on the bispectrum. Being an $\ell$-dependent function it can separate different scale-dependent contributions to the bispectrum and thus can be valuable in separating systematics from true signal. An implementation of skew-spectrum in the context of weak-lensing analysis will be presented elsewhere (Munshi et al. 2019; in preparation).

6 http://sci.esa.int/euclid/

7 http://www.lsst.org/llst home.shtml

8 http://www.sdss3.org/surveys/boss.php

9 http://wigglez.swin.edu.au/ 


\section{The Weak Lensing Bispectrum Induced By Gravity}
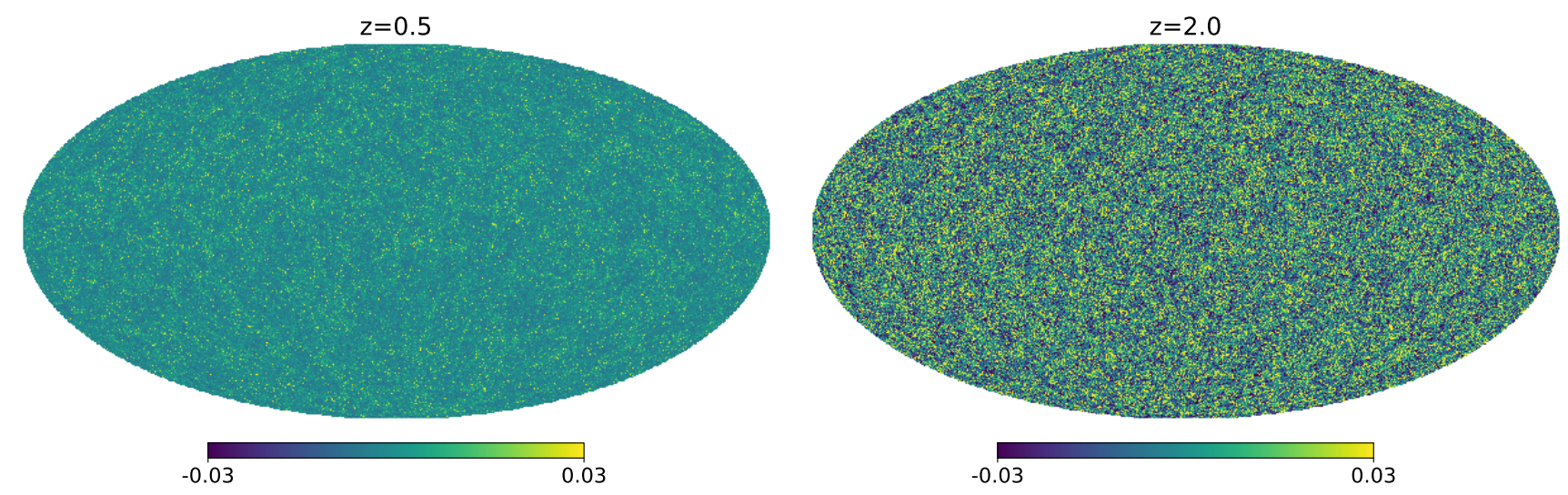

Figure 1. Examples of simulated $\kappa$ maps used in our study. The left panel corresponds to $z_{s}=0.5$ while the right panel corresponds to $z_{s}=2.0$. The maps were generated at a resolution of $N_{\text {side }}=4096$. See $\$ 3$ for more detail discussion about construction of maps used in our study.

The modal decomposition (Fergusson, Ligouri \& Shellard 2016) is a generalisation of KSW estimator and it relies on the expansion of the target or theoretical bispectrum (template) as well as the bispectrum of the map in a basis function. A general basis function can be tackled in this method and separability is not a requirement.

\subsection{Angular Bispectrum}

In this Section we provide a brief review of the existing literature and collate the available results that will be later used in this paper. The weak lensing convergence (Munshi et al. 2008), $\kappa^{\alpha}$ and its harmonic coefficients, $\kappa_{\ell m}^{\alpha}$, are related through the following forward and reverse harmonic transformations and are expressed in terms of the spherical harmonics of degree $\ell$ and order $m$ : $Y_{\ell m}\left(\widehat{\boldsymbol{\Omega}}_{p}\right)$ defined over a discretized sphere as:

$\kappa_{\ell m}^{\alpha}=\sum_{p} \kappa^{\alpha}\left(\widehat{\boldsymbol{\Omega}}_{p}\right) Y_{\ell m}^{\star}\left(\widehat{\boldsymbol{\Omega}}_{p}\right) \triangle \widehat{\boldsymbol{\Omega}}_{p} ; \quad \kappa^{\alpha}\left(\widehat{\boldsymbol{\Omega}}_{p}\right)=\sum_{\ell m} \kappa_{\ell m}^{\alpha} Y_{\ell m}\left(\widehat{\boldsymbol{\Omega}}_{p}\right)$

The Greek index $\alpha$ labels tomographic bins. Here the unit vector $\widehat{\boldsymbol{\Omega}}_{p}$ describes the angular position of the $p$-th pixel, ${ }^{*}$ represents complex conjugation and $\triangle \widehat{\boldsymbol{\Omega}}_{p}$ denotes the pixel area (assumed to be the same for all pixels). The three-point correlation function of the spherical harmonic coefficients also known as the angular bispectrum is defined through the following expression; see e.g. Bartolo et al. (2004) for a review

$$
\begin{aligned}
& \left\langle\kappa_{\ell_{1} m_{1}}^{\alpha} \kappa_{\ell_{2} m_{2}}^{\beta} \kappa_{\ell_{3} m_{3}}^{\gamma}\right\rangle=B_{\ell_{1} \ell_{2} \ell_{3}}^{\alpha \beta \gamma}\left(\begin{array}{ccc}
\ell_{1} & \ell_{2} & \ell_{3} \\
m_{1} & m_{2} & m_{3}
\end{array}\right) ; \\
& B_{\ell_{1} \ell_{2} \ell_{3}}^{\alpha \beta \gamma}=\sum_{m_{1} m_{2} m_{3}}\left(\begin{array}{ccc}
\ell_{1} & \ell_{2} & \ell_{3} \\
m_{1} & m_{2} & m_{3}
\end{array}\right)\left\langle\kappa_{\ell_{1} m_{1}}^{\alpha} \kappa_{\ell_{2} m_{2}}^{\beta} \kappa_{\ell_{3} m_{3}}^{\gamma}\right\rangle .
\end{aligned}
$$

The angular brackets represent ensemble averaging. This form preserves the the rotational invariance of the three-point correlation function in the harmonic domain. The quantity in parentheses is the Wigner $3 \mathrm{j}$-symbol, which is nonzero only for triplets $\left(\ell_{1}, \ell_{2}, \ell_{3}\right)$ satisfying the triangularity condition $\left|\ell_{1}-\ell_{2}\right| \leq \ell_{3} \leq \ell_{1}+\ell_{2}$ and the condition that the sum $\ell_{1}+\ell_{2}+\ell_{3}$ is even, ensuring the parity invariance of the bispectrum (we will ignore any parity violating physics). Parity invariance imposes a selection rule that ensures underlying invariance of the field under spatial inversion. The analysis here can be extended to directly compute the bispectrum from shear $\gamma$ maps that would include both "electric" E and "magnetic" B modes. The parity violating contributions to bispectrum can be obtained by constructing a bispectrum that includes both $\mathrm{E}$ and B modes. This can be used to detect any possible parity violating physics as well as other systematic effects.

The reduced bispectrum $b_{\ell_{1} \ell_{2} \ell_{3}}$ is typically defined through the following relation by introducing a geometric factor $h_{\ell_{1} \ell_{2} \ell_{3}}$ that only depends on $\ell$ and not on the convergence map $\kappa$ :

$b_{\ell_{1} \ell_{2} \ell_{3}}^{\alpha \beta \gamma}=h_{\ell_{1} \ell_{2} \ell_{3}}^{-1} B_{\ell_{1} \ell_{2} \ell_{3}}^{\alpha \beta \gamma} ; \quad h_{l_{1} l_{2} l_{3}}^{2}=\frac{\Sigma_{\ell_{1}} \Sigma_{\ell_{2}} \Sigma_{\ell_{3}}}{4 \pi}\left(\begin{array}{ccc}\ell_{1} & \ell_{2} & \ell_{3} \\ 0 & 0 & 0\end{array}\right)^{2} ; \quad \Sigma_{\ell_{i}}=2 \ell_{i}+1$.

The reduced bispectrum $b_{\ell_{1} \ell_{2} \ell_{3}}$ was introduced by Komatsu \& Spergel (2001), see Babich, Creminelli, Zaldarriaga (2004) for an elaborate 


\section{$4 \quad$ Munshi et al.}

discussion, it is useful in establishing the all-sky and flat-sky correspondence. The factor $h_{\ell_{1} \ell_{2} \ell_{3}}$ represents the geometrical factors that depend on the harmonics $\ell$. We will write Eq. $2 \mathrm{~b}$ using the formal expression that will be used below to define the estimator:

$B_{\ell_{1} \ell_{2} \ell_{3}}^{\alpha \beta \gamma}=\left\langle\int d \widehat{\boldsymbol{\Omega}} \mathcal{L}_{\ell_{1}}^{\alpha}(\widehat{\boldsymbol{\Omega}}) \mathcal{L}_{\ell_{2}}^{\beta}(\widehat{\boldsymbol{\Omega}}) \mathcal{L}_{\ell_{3}}^{\gamma}(\widehat{\boldsymbol{\Omega}})\right\rangle ; \quad \mathcal{L}_{\ell_{i}}^{\alpha}(\widehat{\boldsymbol{\Omega}})=\sum_{m_{i}=-\ell_{i}}^{\ell_{i}} \kappa_{\ell_{i} m_{i}}^{\alpha} Y_{\ell_{i} m_{i}}(\widehat{\boldsymbol{\Omega}})$.

The fields $\mathcal{L}_{\ell_{i}}^{\alpha}$ are constructed using maximally filtered convergence or $\kappa$ fields by keeping only one harmonic mode. A binned version which we will implement is defined by constructing the $\mathcal{L}_{b_{i}}^{\alpha}$ which uses the coarse-grained harmonics defined in a bin. Binning reduces the scatter in the estimator; however, unbinned estimates can be used as well for parameter estimations. The binning will mix various shapes that we will discuss later.

$B_{b_{1} b_{2} b_{3}}^{\alpha \beta \gamma}=\left\langle\int d \widehat{\boldsymbol{\Omega}} \mathcal{L}_{b_{1}}^{\alpha}(\widehat{\boldsymbol{\Omega}}) \mathcal{L}_{b_{2}}^{\beta}(\widehat{\boldsymbol{\Omega}}) \mathcal{L}_{b_{3}}^{\gamma}(\widehat{\boldsymbol{\Omega}})\right\rangle ; \quad \mathcal{L}_{b_{i}}^{\alpha}(\widehat{\boldsymbol{\Omega}})=\sum_{\ell \in b_{i}} \sum_{m=-\ell}^{\ell} \kappa_{\ell m}^{\alpha} Y_{\ell m}(\widehat{\boldsymbol{\Omega}})$.

The angular brackets represent ensemble averaging. In the following, we will discuss the coarse-grained estimator for the reduced bispectrum. Notice that in addition to cross-correlating different tomographic bins, the estimator can also be used to cross-correlate external fields including e.g., the $y$-parameter maps from thermal Sunyaev-Zel'dovich (tSZ) effect. A simple expression for the covariance can be constructed in the noise dominated regime:

$$
\begin{aligned}
& \left\langle\hat{B}_{\ell_{1} \ell_{2} \ell_{3}}^{\alpha \beta \gamma} \hat{B}_{\ell_{4} \ell_{5} \ell_{6}}^{\mu \nu \eta}\right\rangle=h_{\ell_{1} \ell_{2} \ell_{3}}^{2} \mathcal{C}_{\ell_{1}}^{\alpha \mu} \mathcal{C}_{\ell_{2}}^{\beta \nu} \mathcal{C}_{\ell_{3}}^{\gamma \eta} \delta_{\ell_{1} \ell_{4}} \delta_{\ell_{2} \ell_{5}} \delta_{\ell_{3} \ell_{6}}+\text { cyc.perm. } \\
& \sigma^{2}\left(B_{\ell_{1} \ell_{2} \ell_{3}}^{\alpha \beta \gamma}\right)=\sum_{\ell_{1} \in \Delta_{1}} \sum_{\ell_{2} \in \Delta_{2}} \sum_{\ell_{3} \in \Delta_{3}} h_{\ell_{1} \ell_{2} \ell_{3}}^{2} \mathcal{C}_{\ell_{1}}^{\alpha \beta} \mathcal{C}_{\ell_{2}}^{\beta \gamma} \mathcal{C}_{\ell_{3}}^{\gamma \alpha}+\text { cyc.perm. }
\end{aligned}
$$

The expressions "cyc. perm." above represent a cyclic permutation of the superscripts (greek-indices) which represents individual tomographic bins. The angular cross-spectra of two different bins are represented by $\mathcal{C}_{\ell}^{\alpha \beta}=\left\langle\kappa_{\ell m}^{\alpha} \kappa_{\ell m}^{\beta *}\right\rangle$. In case of a partial-sky coverage originating from an observational mask the linear term needs to be subtracted to make the estimator unbiased (Munshi \& Heavens 2010):

$B_{\ell_{1} \ell_{2} \ell_{3}}^{\alpha \beta \gamma(\operatorname{lin})}=\sum_{p}^{N_{\text {pix }}}\left[\mathcal{L}_{\ell_{1}}^{\alpha}\left(\widehat{\boldsymbol{\Omega}}_{p}\right)\left\langle\mathcal{L}_{\ell_{2}}^{\beta}\left(\widehat{\boldsymbol{\Omega}}_{p}\right) \mathcal{L}_{\ell_{3}}^{\gamma}\left(\widehat{\boldsymbol{\Omega}}_{p}\right)\right\rangle+\mathcal{L}_{\ell_{2}}^{\beta}\left(\widehat{\boldsymbol{\Omega}}_{p}\right)\left\langle\mathcal{L}_{\ell_{1}}^{\alpha}\left(\widehat{\boldsymbol{\Omega}}_{p}\right) \mathcal{L}_{\ell_{3}}^{\gamma}\left(\widehat{\boldsymbol{\Omega}}_{p}\right)\right\rangle+\mathcal{L}_{\ell_{3}}^{\gamma}\left(\widehat{\boldsymbol{\Omega}}_{p}\right)\left\langle\mathcal{L}_{\ell_{1}}^{\alpha}\left(\widehat{\boldsymbol{\Omega}}_{p}\right) \mathcal{L}_{\ell_{2}}^{\beta}\left(\widehat{\boldsymbol{\Omega}}_{p}\right)\right\rangle\right]$.

Here $N_{\text {pix }}$ is the number of pixel and the discrete summation (which replaces integrals) represents a pixelization scheme. The ensemble averaging is computed by using many Gaussian realisations with the same power spectrum $\mathcal{C}_{\ell}$ and the resulting unbiased estimator $\hat{B}$ can now be constructed from the pseudo estimator $\tilde{B}$ :

$\widehat{B}_{\ell_{1} \ell_{2} \ell_{3}}^{\alpha \beta \gamma}=\widetilde{B}_{\ell_{1} \ell_{2} \ell_{3}}^{\alpha \beta \gamma}-B_{\ell_{1} \ell_{2} \ell_{3}}^{\alpha \beta \gamma(\text { lin })}$

The pseudo estimator $\tilde{B}$ is constructed by restricting the integration in Eq. 3 and Eq. 4 to the observed region of the sky. Here, $\widehat{B}_{\ell_{1} \ell_{2} \ell_{3}}^{\alpha \beta \gamma}$ represents the all-sky estimator which is constructed from a partial sky measurement represented by $\widetilde{B}_{\ell_{1} \ell_{2} \ell_{3}}^{\alpha \beta \gamma}$ and corrections due to partial sky coverage is encoded in the term $B_{\ell_{1} \ell_{2} \ell_{3}}^{\alpha \beta \gamma(\text { lin) }}$ defined in Eq. 7 .

\subsection{Estimator}

Using the definitions in the previous section we outline the estimator here that will be used for analysing the simulations. The reduced bispectrum $b_{\ell_{1} \ell_{2} \ell_{3}}$ can be estimated by the following expression (see (Namikawa et al. 2018) for a detailed derivation).

$b_{\ell_{1} \ell_{2} \ell_{3}}=\frac{1}{h_{\ell_{1} \ell_{2} \ell_{3}}^{2}} \sum_{p}^{N_{\text {pix }}} \mathcal{L}_{1}^{\alpha}\left(\widehat{\boldsymbol{\Omega}}_{p}\right) \mathcal{L}_{2}^{\beta}\left(\widehat{\boldsymbol{\Omega}}_{p}\right) \mathcal{L}_{3}^{\gamma}\left(\widehat{\boldsymbol{\Omega}}_{p}\right) \approx \sqrt{4 \pi} \frac{\sum_{p}^{N_{\text {pix }}} \mathcal{L}_{\ell_{1}}^{\alpha}\left(\widehat{\boldsymbol{\Omega}}_{p}\right) \mathcal{L}_{\ell_{2}}^{\beta}\left(\widehat{\boldsymbol{\Omega}}_{p}\right) \mathcal{L}_{\ell_{3}}^{\gamma}\left(\widehat{\boldsymbol{\Omega}}_{p}\right)}{\sum_{p}^{N_{\text {pix }}} h_{\ell_{1}}\left(\widehat{\boldsymbol{\Omega}}_{p}\right) h_{\ell_{2}}\left(\widehat{\boldsymbol{\Omega}}_{p}\right) h_{\ell_{3}}\left(\widehat{\boldsymbol{\Omega}}_{p}\right)}$

The first equality uses the expression of $b_{\ell_{1} \ell_{2} \ell_{3}}^{\alpha \beta \gamma}$ in terms of $B_{\ell_{1} \ell_{2} \ell_{3}}^{\alpha \beta \gamma}$ as given in Eq. 3 and uses the definition of $\mathcal{L}_{\ell_{i}}^{\alpha}$ in Eq. 4 . $B_{\ell_{1} \ell_{2} \ell_{3}}^{\alpha \beta \gamma}$. The individual $\mathcal{L}$ maps are constructed using modes estimated from maps as described in Eq. 5. The construction of the denominator can be done either using the standard textbook (Edmonds 1968) expression or using similar technique used for the numerator. We start by noticing the fact that $h_{\ell_{1} \ell_{2} \ell_{3}}^{2}$ defined in Eq. 3 . can be expressed as a Gaunt Integral as follows:

$h_{\ell_{1} \ell_{2} \ell_{3}}^{2}=\sqrt{\frac{\left(2 \ell_{1}+1\right)\left(2 \ell_{2}+1\right)\left(2 \ell_{3}+1\right)}{4 \pi}} \int Y_{\ell_{1} 0}(\widehat{\boldsymbol{\Omega}}) Y_{\ell_{2} 0}(\widehat{\boldsymbol{\Omega}}) Y_{\ell_{3} 0}(\widehat{\boldsymbol{\Omega}}) d \widehat{\boldsymbol{\Omega}}$. 
Next, we define the functions $h_{i}\left(\widehat{\boldsymbol{\Omega}}_{p}\right)$ as follows and use a discrete sum to replace the integral:

$h_{\ell_{1} \ell_{2} \ell_{3}}^{2}=\frac{1}{\sqrt{4 \pi}} \sum_{p}^{N_{\text {pix }}} h_{1}\left(\widehat{\boldsymbol{\Omega}}_{p}\right) h_{2}\left(\widehat{\boldsymbol{\Omega}}_{p}\right) h_{3}\left(\widehat{\boldsymbol{\Omega}}_{p}\right) ; \quad h_{\ell_{i}}\left(\widehat{\boldsymbol{\Omega}}_{p}\right)=\sum_{\ell} \sqrt{(2 \ell+1)} Y_{\ell_{i} 0}\left(\widehat{\boldsymbol{\Omega}}_{p}\right)$.

This factor does not depend on the source-redshift or convergence power spectrum and has a purely geometric origin. To reduce the computational cost as well as the scatter we provide the bin estimates instead of the mode-by-mode result. Notice that binning mixes different types of bispectrum as all possible modes in a given bin contribute. However, this effect can be modelled theoretically before comparing with data. Reducing the size of the bins can effectively control such contamination. Previous study by Namikawa et al. (2018) has found the effect of binning to be significant for all shapes but comparatively less significant for the equilateral case. The binning can be introduced through the following expression:

$b_{b_{1} b_{2} b_{3}}^{\alpha \beta \gamma}=\frac{\sum_{\ell_{i} \in b_{i}} h_{\ell_{1} \ell_{2} \ell_{3}}^{2} b_{\ell_{1} \ell_{2} \ell_{3}}^{\alpha \beta \gamma}}{\sum_{\ell_{i} \in b_{i}} h_{\ell_{1} \ell_{2} \ell_{3}}^{2}}$.

As we mentioned before, the above estimator can also be generalised to analyse directly the shear maps by decomposing them into electric $\mathrm{E}$ and magnetic B modes. The linear terms appearing due to partial sky coverage will be introduced later in this section.

Typically two different methods are pursued in the detection of non-Gaussianity. The first method uses a template for the detection relying on a matched-filter technique. In this case, the spherical harmonics coefficients are convolved with weights to make them near optimal. Such methods work in the weakly non-Gaussian case. In case of lensing, non-Gaussianity is more dominant and optimality is less of an issue as signal-to-noise is rather high. We choose a non-parametric estimator (also called a binned estimator) which is sub-optimal but that does not rely on a theoretical model.

\subsection{Convergence Bispectrum and Matter Bispectrum}

In this section we express the weak lensing bispectrum $\mathcal{B}$ as a line of sight integration of bispectrum $B_{\delta}$ associated with the density contrast $\delta$. The gravitational deflection potential $\phi$, which is related to the convergence $\kappa$, can be expressed as a line of sight integration of Weyl potential $\Phi$ as follows:

$$
\phi(\widehat{\boldsymbol{\Omega}})=-2 \int_{0}^{\chi_{s}} d \chi W\left(\chi, \chi_{s}\right) \Phi(\chi, \widehat{\boldsymbol{\Omega}}) ; \quad W\left(\chi, \chi_{s}\right)=\frac{\chi_{s}-\chi}{\chi_{\chi_{s}}} \Theta\left(\chi_{s}-\chi\right) ; \quad \kappa_{\ell m}=\frac{1}{2} \ell(\ell+1) \phi_{\ell m} .
$$

Here, $W\left(\chi, \chi_{\star}\right)$ represents the lensing kernel and $\chi$ is the radial comoving distance and $\Theta$ is the unit step function. The comoving radial distance to the source plane is denoted as $\chi_{s}$. Using the flat-sky approximation the convergence bispectrum $\mathcal{B}_{\ell_{1} \ell_{2} \ell_{3}}$ can be related to the underlying matter bispectrum $B_{\delta}$ through the following line-of-sight integration:

$\mathcal{B}_{\ell_{1} \ell_{2} \ell_{3}}=\int_{0}^{\chi_{s}} d \chi\left[\frac{3 \Omega_{\mathrm{M}} H_{0}^{2}}{2 a(\chi)}\right]^{3} \chi^{2} W^{3}\left(\chi, \chi_{s}\right) B_{\delta}\left(\frac{\ell_{1}}{\chi}, \frac{\ell_{2}}{\chi}, \frac{\ell_{3}}{\chi}, \chi\right) ; \quad k_{i}=\frac{\ell_{i}}{\chi}$.

The flat-sky bispectrum is defined in terms of the 2D Fourier coefficients $\kappa(\mathbf{l})$ :

$\left\langle\kappa\left(\mathbf{l}_{1}\right) \kappa\left(\mathbf{l}_{2}\right) \kappa\left(\mathbf{l}_{3}\right)\right\rangle=(2 \pi)^{2} \delta_{2 \mathrm{D}}\left(\mathbf{l}_{1}+\mathbf{l}_{2}+\mathbf{l}_{3}\right) b\left(\mathbf{l}_{1}, \mathbf{l}_{2}, \mathbf{l}_{3}\right)$.

The reduced bispectrum satisfies the relationship $b_{\ell_{1} \ell_{2} \ell_{3}} \approx b\left(\mathbf{l}_{1}, \mathbf{l}_{2}, \mathbf{l}_{3}\right)$ (Bartolo et al. 2004). Our analytical predictions are derived using the flat-sky approximation, though, the numerical results are derived using all-sky simulation we expect that for $\ell>100$ these two results should match; see e.g. Kitching et al. (2017) for related discussions in the context of power spectrum. The gravity induced matter bispectrum $B_{\delta}\left(\mathbf{k}_{1}, \mathbf{k}_{2}, \mathbf{k}_{3}, \chi\right)$ is often modelled using standard tree-level perturbation theory in the quasilinear regime. We use a fitting function for $B_{\delta}$ in our computation of $\mathcal{B}$ using Eq. 13 which is described in Appendix-s A generalization to 3D bispectrum is presented in in Appendix- $\mathrm{B}$

\subsection{Shapes of Weak Lensing Convergence Bispectrum}

The bispectrum represents three-way interaction of three different wave vectors in 3D or harmonic multipoles in spherical sky. To guarantee translational invariance in the case of $3 \mathrm{D}$ and rotational invariance on the sphere the vectors should form a closed triangle. Various triangular configurations have been studied in great detail in the context of inflationary models and they can be related to a specific inflationary mechanism, e.g., the squeezed model is related to the local form of non-Gaussianity (Salopek \& Bond 1990. 1991, Gangui et al. 1994), and the equilateral form of primordial non-Gaussianity appears in the inflationary models with a non-canonical kinetic term (Alishahiha et al.2004 Chen, Easther, Lim 2007, Cheung et al. 2008, Creminelli et al. 2006).

Concerning the input of large scale structure formation scenarios, the various shapes can also give clues on the gravitational dynamics 

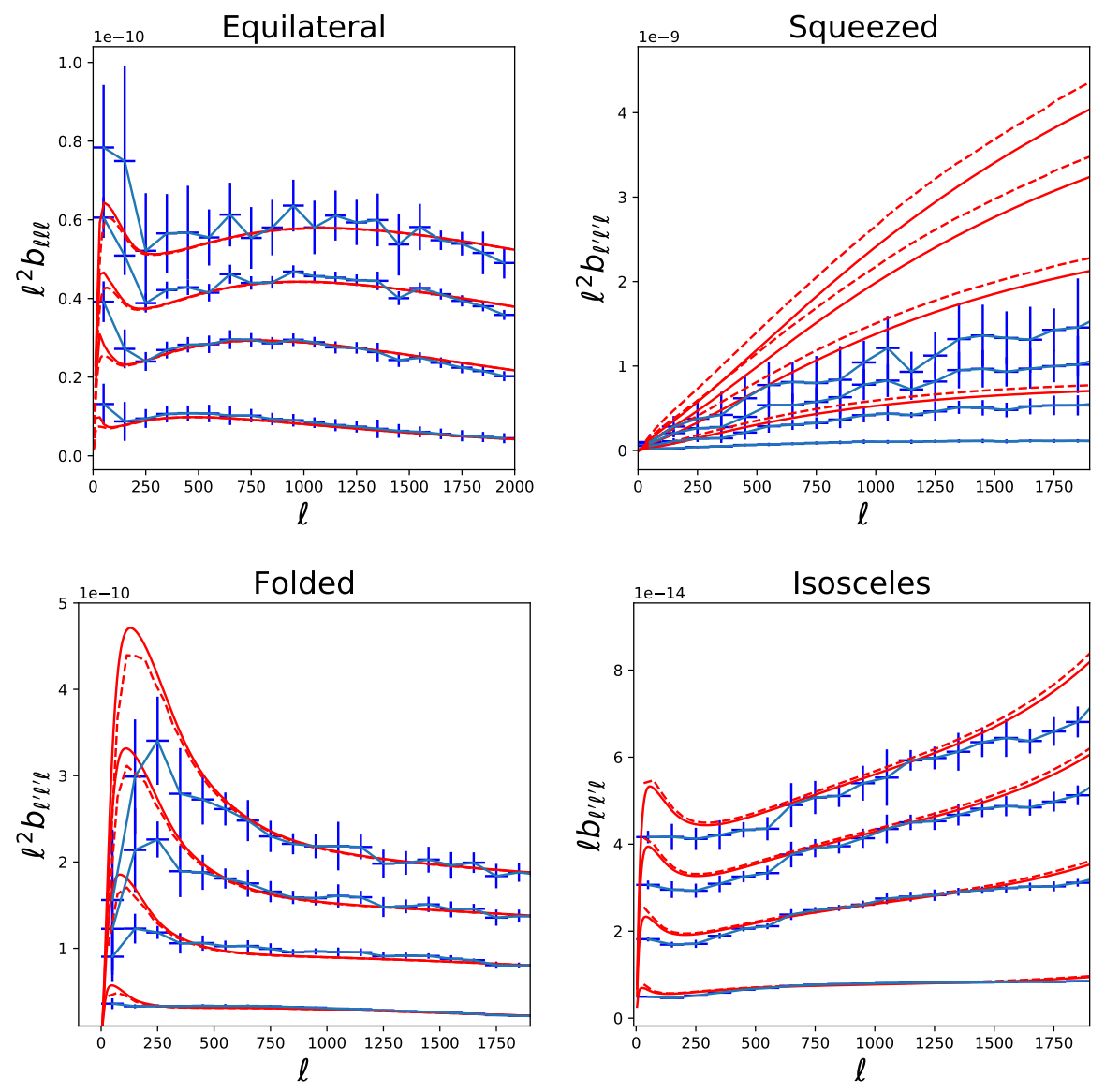

Figure 2. The reduced bispectrum $b_{\ell_{1} \ell_{2} \ell_{3}}$ measured in simulations for four different shapes are compared against theoretical predictions. The upper left-panel corresponds to the equilateral configuration $\ell_{1}=\ell_{2}=\ell_{3}=\ell$ and depicts $\ell^{2} b_{\ell \ell \ell}$ as a function of $\ell$. The sources are placed at different redshifts; from bottom to top they correspond respectively to $z_{s}=0.5,1.0,1.5$ and 2.0. The theoretical predictions are computed using the fitting function given in (Gil-Marin et al.2011). The maps used for this analysis were constructed at $N_{\text {side }}=4096$. No noise or mask were included. For the squeezed case (upper right-panel) the bispectrum $b_{\ell^{\prime} \ell^{\prime} \ell}$ is shown for $\ell_{1}=\ell^{\prime}=50$ and $\ell_{2}=\ell_{3}=\ell$. The error-bars were computed using ten realisations for each redshift. We use a total of 20 bins to cover the range of multipoles. The solid lines in each panel correspond to theoretical predictions computed using the fit in Eq. (A1/A3c). The dashed lines include the effect of binning. The expressions for the binned bispectrum are presented in Eq.111. The lower left-panel shows the folded configuration while the lower right-panel depicts the isosceles configuration. For the isosceles case we take $\ell_{1}=\ell$ and $\ell_{2}=\ell_{3}=1000$. For the folded case we have $\ell_{1}=2 \ell_{2}=2 \ell_{3}=\ell$ and we plot $\ell b_{\ell^{\prime} \ell^{\prime} \ell}$ as a function of $\ell$. Our results are derived using flat-sky approximations.

at work to create the resulting convergence maps. For the equilateral case the length of three sides of the triangle are equal and in real space this bispectrum can represent possibilities as dictated by the signature of the bispectrum. A positive amplitude for the reduced bispectrum will correspond to compact collapsed over dense regions surrounded by underdense regions. Whereas the negative amplitude corresponds to underdense regions surrounded by over-dense objects. The positive bispectrum will correspond to positive skewness (representing long tail of positive $\kappa$ values) and similarly a negative bispectrum will represent negative skewness that correspond to long tail of negative $\kappa$ values in probability distribution function of $\kappa$. The formation of large scale structure formation is known to induce a positive skewness and hence a positive amplitude for the reduced bispectrum.

Exact equilateral configurations are less likely just as numerical simulations in real space do not often produce exact spherically collapsed over-dense regions. The elliptical or elongated over-dense regions are represented by folded configuration of bispectrum. In the limiting case these can take the shape of filaments.

The squeezed configuration corresponds to the situation when one wave vector is much smaller compared to the other two. In the extreme squeezed limit when one of the wave vectors approaches zero, the other two wave vectors are equal and opposite to each other. The squeezed 
configuration thus can be understood as a response to a long wavelength modulation of the local power spectrum. This response function approach (Berreira \& Schmidt 2017) have recently been studied and investigated in detail by many authors in a scenario also known as the separate universe formalism (Baldauf et al. 2016, Li, Hu, Takada 2014). This method was recently studied in the context of weak lensing convergence maps using a different approach that we use here by (Munshi et al. 2019). For a Gaussian random field, the coupling of local fluctuations to long wavelength modulation is zero. For a positive bispectrum, long term fluctuations enhance the small scale collapse. The squeezed limit has also been studied in diverse cosmological context, e.g., in the case of CMB lensing, galaxy clustering as well as Lyman- $\alpha$ absorption studies; see e.g. (Chiang et al. 2015, Chinag et al. 2017, Chiang \& Slosar 2018, Munshi et al. 2019) and references therein. The study of squeezed limits are important from another aspect. In recent studies consistency relations were obtained in the squeezed limit which can be used as a diagnostics to test any violation from Gaussian initial conditions or modification of gravity. The test of such consistency relations can be performed using weak lensing observations that can give a rare glimpse of non-perturbative regime of gravitational clustering (Kehagias \& Riotto 2001).

Clearly the one-to-one correspondence of $3 \mathrm{D}$ shapes and their 2D counterparts is lost due to projection. The numerical results for these configurations will be presented in $\$$

\section{SIMULATIONS}

We use the all-sky weak-lensing maps generated by (Takahashi et al. $\left.2017\right|^{10}$ These maps were generated using ray-tracing through N-body simulations using multiple lens planes and to generate convergence $\kappa$ as well as shear $\gamma$ maps. The source redshifts used were in the range $z_{s}=0.05-5.30$ at an interval $\Delta \chi=150 h^{-1} \mathrm{Mpc}$ (corresponding to $\Delta z_{s}=0.05$ at the nearby universe). The simulations also include maps generated for sources placed at last scattering surface for CMB lensing studies. The simulations do not assume the Born approximations and do include post-Born corrections and its higher-order analogs to an arbitrary order. However, such corrections are expected to be negligible for the low source redshift that we are interested here (Namikawa et al. 2018). Many checks were conducted using these maps and were found to be in good agreement with theoretical predictions. For a given redshift, sets of maps were generated using different resolution in HEALPix ${ }^{11}$ format (Gorski et al. 2016) which uses equal area pixelization scheme. The number of pixels $N_{\text {pix }}=12 N_{\text {side }}^{2}$ is defined in terms of the resolution parameter $N_{\text {side. }}$. Detail test of electric/magnetic (E/B) decomposition of shear maps and construction of $\kappa$ maps can be found in (Takahashi et al. 2017). For our purpose we use the $\kappa$ maps generated at $N_{\text {side }}=4096$. These maps have been cross checked using higher resolution maps constructed at $N_{\text {side }}=8192,16384$ and were found to be in good agreement with theoretical predictions up to the angular harmonics $\ell \leq 3600$. The corresponding angular resolutions are $0.86^{\prime}, 0.43^{\prime}$ and $0.21^{\prime}$ for $N_{\text {side }}=4096,8192$ and 16384 respectively. In our study we will be restricting us to $\ell \leq 2048$. Beyond this limit it is expected that baryonic feedback may play increasingly dominant role (Weiss et al. 2019). These maps were recently used to analyse the bispectrum in a recent publication but in the context of CMB lensing (Namikawa et al. 2018). In Figure-1 we show examples of maps used in our study.

These simulations assume a $\Lambda \mathrm{CDM}$ background cosmology with the following set of cosmological parameters $\Omega_{\mathrm{CDM}}=0.233, \Omega_{b}=$ 0.046 and $\Omega_{\mathrm{M}}=\Omega_{\mathrm{CDM}}+\Omega_{b}=0.279$. The Hubble parameter was assumed to be $h=0.7$, the amplitude of density flcutuation $\sigma_{8}=0.82$ and the spectral index $n_{s}=0.97$.

\section{RESULTS}

Bispectrum and other higher-order multispectra will be detected in ongoing and future weak lensing surveys with a high $\mathrm{S} / \mathrm{N}$ ratio. In this paper we compare the theoretical modelling of bispectrum with high resolution all-sky numerical simulations. In principle all possible configurations of the bispectrum should be considered. This is computationally demanding so, we have concentrated on specific configurations of the bispectrum. In our numerical investigation we have considered the following representative shapes for the bispectrum: (A) Squeezed: $\ell_{1}=50, \ell_{2}=\ell_{3}=\ell \quad$ (B) Equilateral: $\ell_{1}=\ell_{2}=\ell_{3}=\ell$ (C) Isoceles: $\ell_{1}=\ell, \ell_{2}=\ell_{3}=1000$ and $\quad$ (D) Folded: $\ell_{1}=2 \ell_{2}=2 \ell_{3}=\ell$. We have restricted our comparison to $\ell<2048$ modes as it is expected that the higher-order multipoles will be affected by baryonic feedback that are difficult to model accurately. For each model we have considered four tomogrphaic bins $z_{s}=0.5,1.0,1.5,2.0$. The source redshift $z_{s}$ increases from bottom to top in each panel for various curves shown in Figure-2

The results for equilateral configurations are shown in Figure-2(upper left-panel). The numerical results agree reasonably well with the theoretical predictions based on commonly used fitting functions for $\ell>200$, at least for $\ell<2048$. This is consistent with the general trend of results reported in (Namikawa et al. 2018). This deviation of numerical results at lower $\ell$ s from theoretical predictions is visible in all of the models we have studied and is likely to be related to the use of flat-sky approximation in our theoretical derivations. Corrections, to flat-sky

10 http://cosmo.phys.hirosaki-u.ac.jp/takahasi/allsky_raytracing/

11 https://healpix.jpl.nasa.gov/ 
results can be included in a systematic manner (Kitching et al.|2017). The results of comparison for the squeezed model is presented in (upper right-panel). We see maximum departure from theoretical predictions in case of squeezed configuration where the theoretical predictions can be higher by a factor of two compared to the results obtained from simulations. The Figure-2 depicts the folded (lower left-panel) and the isosceles (lower right-panel) case. In case of the folded model we find significant departure from theoretical predictions at $\ell<500$. The theoretical model can predict the results from simulations reasonably well. However, the small $\ell$ discrepancy is far more pronounced than other models. The isosceles case only matches with theoretical predictions for $\ell=1024$ where the configuration is actually close to the equilateral case.

While corrections to the flat-sky approximation may explain suppression of the low $\ell$ results in case of equilateral model, the finite volume corrections can not be ignored for other cases. The bispectrum is a nonlinear statistic. The effect of finite volume is well studied in the real-space at the level of one point statistics, e.g., cumulants and their correlators. These effects are also well documented for estimation of power spectrum also known as the integral constraint - see e.g. Bernardeau et al (2002). However, similar studies are lacking for the bispectrum which is proportional to the product of two power spectra probing different scales and hence are expected to be more affected by such an effect. This is also true in real space where e.g. skewness and other higher-order moments are more affected by the finite volume effect than the variance. The finite volume effect is more pronounced in the highly nonlinear regime. In equilateral case only one scale is involved making its interpretation comparatively simpler. For other three cases, different levels of nonlinearities are probed by the different wave numbers of the bispectrum. In any case, the fact that finite volume correction is a factor in our results, can easily be seen from Figure-2 The $\mathrm{CMB}$ lensing on the other hand, where the source is at a rather high redshift $z_{s} \approx 1100$, probes the quasi-linear regime thus reducing the effect of finite volume corrections. The corrections to the flat-sky expressions are also less prominent for a high source redshift. A broader binning can reduce the discrepancy between theory and simulations that we have noted but at the cost of degrading the cosmological information content of the spectrum..

The accuracy of the fitting function we use may not be adequate to describe the range of redshift and harmonic modes we have studied. An improved fitting function for the bispectrum, that is currently underway will be presented elsewhere (Takahashi et al. 2019, in preparation).

\section{DISCUSSION}

The gravity induced bispectrum, arising from non-Gaussianity in weak lensing maps, can help to tighten the constraints on cosmological parameters. We have tested available theoretical fitting functions accuracy to reproduce numerical results. The lensing bispectrum as a possible probe for modified gravity theories as well as neutrino masses will be a natural future step.

Impact of modified gravity theories on CMB lensing induced bispectrum was studied in (Namikawa et al. 2018) to some extent. Theoretical modelling of bispectrum in modified gravity scenarios is more challenging. Extensions of the perturbative approach was considered in (Bose \& Taruya 2018, Namikawa, Bouchet \& Taruya 2018) by introducing more freedom to the kernels and validating them using numerical simulations. Such approaches and others including halo model predictions have already been proposed with varying degree of success for two main type of modified gravity theories, i.e., (A) models with Vainshtein-screening mechanism which includes the DGP model as well as the Hordenski (Hordenski 1974) and beyond Hordenski theories (Gleyzes et al. 2015a b; Langois \& Noui 2016) and (B) the models with Chameleon-screening that includes the Hu-Sawicki $f(\mathrm{R})$ model (Hu \& Sawicki 2016). In the DGP model (Dvali, Gabadadze, Porati 2000) the bispectrum from simulations can be reproduced using the GR expression by suitably modifying the power spectrum. The situation is somewhat more complicated for $f(R)$ theories. However, the fitting used for GR needs first to be standardised more accurately. Only then can the deviations from them can be studied meaningfully. The numerical modelling is more important at small scales where analytical results start to fail. In future it will also be interesting to consider the effect of neutrino mass on bispectrum when simulated all-sky lensing maps for such cosmologies will be available (Liu et al. 2018, Coulton et al. 2018).

In addition to studying various shapes of weak lensing bispectrum we have extended the framework in several directions. We have computed the covariance of our estimator in the noise dominated regime in the weakly non-Gaussian limit. We have shown how the estimator can include tomographic bins. Using spherical Fourier-Bessel (sFB) decomposition, we have extended the estimator to 3D. Inclusion of weights to make the estimator near-optimal is described in the limit of vanishing non-Gaussianity, i.e., in the noise dominated regime. We also provide a simplistic estimate of the covariance. The extension to next higher-order namely to the level of the reduced trispectrum is outlined in appendix-C

\section{REFERENCES}

T. Abbott, F. B. Abdalla, S. Allam, et al., The Dark Energy Survey Collaboration, 2016, Phys. Rev. D, 94, 022001 arXiv/1507.0552] M. Alishahiha, E. Silverstein, T. Tong, 2004, Phys. Rev. D70, 123505 |hep-th/ $0404084 \mid$

D. Babich, P. Creminelli, M. Zaldarriaga, 2004, JCAP, 0408, 009, arXiv/ 0405356 
A. J. Barber, D. Munshi, P. Valageas, 2004, MNRAS, 347, 667 |astro-ph/0 304451 |

T. Baldauf, U. Seljak, L. Senatore, M. Zaldarriaga 2016, JCAP, 09, 007B [arXiv/1511.01465]

N. Bartolo, E. Komatsu, S. Matarrese, A. Riotto, 2004, Phys.Rept., 402, 103, |arXiv/ $0406398 \mid$

A. Barreira, F. Schmidt 2017, JCAP, 06, 053 |arXiv/1703.09212]

F. Bernardeau, S. Colombi, E. Gaztanaga, R. Scoccimarro, 2002, Phys.Rept. 367, 1, [astro-ph/0112551]

B. Bose, A. Taruya, astro-ph/1808.01120|

B. Bose, J. Byun, F. Lacasa, A. M. Dizgah, L. Lombriser [astro-ph/1909.02504]

M. Bucher, B. Racined, B. v. Tente, 2016, JCAP, 05, 055 [arXiv/ 0406398 |

P. G. Castro, A. F. Heavens, T. D. Kitching, 2005, PRD, 72, 023516 |astro-ph/0503479|

X. Chen, R. Easther, E. A. Lim, 2007, JCAP, 0706, 023 [astro-ph/ 0611645 ]

C. Cheung, P. Creminelli, A. L. Fitzpatrick, J. Kaplan, L. Senatore, 2008, JHEP, 0803, 014 |arXiv/ $0709.0293 \mid$

C.-T. Chiang, C. Wagner, A. G. Snchez, F. Schmidt, E. Komatsu, 2015, JCAP, 09, 028 [astro-ph/1504 . 03322$]$

C.-T. Chiang, A. Slosar, 2018, JCAP, 01, 012, |arXiv/1708.07512|

C.-T. Chiang, A. M. Cieplak, F. Schmidt, A. Slosar, 2017, JCAP, 06, 022C [arXiv/1701.03375]

T. Clifton, P. G. Ferreira, A. Padilla, C. Skordis, 2012, Physics Reports 513, 1, 1 |astro-ph/1106.2476|

Creminelli P., Nicolis A., Senatore L., Tegmark M., Zaldarriaga M., 2006, JCAP, 5, 4 |astro-ph/0509029|

W. R. Coulton, J. Liu, M. S. Madhavacheril, V. Bhm, D. N. Spergel, |arXiv/1810.02374]

V. Desjacques, D. Jeong, F. Schmidt |arXiv/1611.09787|

M. J. Drinkwater, R. J. Jurek, C. Blake, et al. 2010, MNRAS, 401, 14, |astro-ph/0911.4246|

G. Dvali, G. Gabadadze, M. Porrati, 2000, Phys. Rev. B, 485, 208, arXiv/1510.06930]

A. R. Edmonds, Angular Momentum in Quantum Mechanics, 2nd ed. rev. printing. Princeton, NJ:Princeton University Press, 1968.

D. J. Eisenstein, D. H. Weinberg, E. Agol, et al., 2011, AJ, 142, 72, astro-ph/1101.1529]

J. R. Fergusson, M. Liguori, E. P. S. Shellard, 2012, PRD, 82, 023502 |arXiv/0912 .5516|

A. Gangui, F. Lucchin, S. Matarrese, S. Mollerach, 1994, ApJ, 430, 447 [astro-ph/9312033]

H. Gil-Marn, C. Wagn.er, F. Fragkoudi, R. Jimenez, L. Verde [arxiv/1111.4477]

J. Gleyzes, D. Langlois, F. Piazza, F. Vernizzi, 2015, JCAP, 2, 018, |arXiv/1408.1952|

J. Gleyzes, D. Langlois, F. Piazza, F. Vernizzi, 2015, Phys. Rev. Lett., 114, 211101, [arXiv/1404.6495]

K. M. Gorski, E. Hivon, A. J. Banday, B. D. Wandelt, F. K. Hansen, M. Reinecke, M. Bartelman, 2005, ApJ, .622, 759 astro-ph/0409513]

G. W. Horndeski, 1974, International Journal of Theoretical Physics, 10, 363.

W. Hu, 2001, PRD, 64, 083005 arXiv/015117

W. Hu, I. Sawicki, 2007, Phys. Rev. D, 76, 064004, |arXiv/0705.1158|

A. Joyce, B. Jain, J. Khoury, M. Trodden, 2015, Physics Reports, 568, 1 |astro-ph/1407.0059|

A. Kehagias, A. Riotto, 2013, Nucl. Phys. B873, $514 \mid \operatorname{arXiv/1302.0130|}$

T. D. Kitching, J. Alsing, A. F. Heavens, R. Jimenez, J. D. McEwen, L. Verde 2017, MNRAS, 469, 2737 [arXiv/1611 . 04954

E. Komatsu, D. N. Spergel, B. D. Wandelt, 2005, ApJ, 634, 14 astro-ph/0305189|

E. Komatsu, B. D. Wandelt, D. N. Spergel, A. J. Banday, K. M. Gorski, 2002, ApJ., 566, 19 [astro-ph/0107605]

E. Komatsu \& D. N. Spergel, Proceedings of the MGIX MM Meeting. Held 2-8 July 2000 Published by World Scientific Publishing Co.

Pte. Ltd., 2002. ISBN 9789812777386, pp. 2009-2010 |astro-ph/0012197|

M. Kilbinger, P. Schneider 2005, A\&A, 442, 69 [astro-ph/0505581]

K. Kuijken, C. Heymans, H. Hildebrandt, et al., 2015, MNRAS, 454, 3500

R. Laureijs, J. Amiaux, S. Arduini, et al. 2011, ESA/SRE(2011)12

J. Lesgourgues, S. Pastor, 2006, Phys.Rept., 429, 307, [astro-ph/1610.02956]

D. Langlois, K. Noui, 2016, JCAP, 2, 034, |arXiv/1510.06930|

A. Lewis, 2011, JCAP, 10, 026 arXiv/1107.5431]

J. Liu, S. Bird., J. M. Z. Matilla, J. C. Hill, Z. Haiman, M. S. Madhavacheril, D. N. Spergel, A. Petri, 2018, JCAP|arXiv/1711.10524

Y. Li, W. Hu, M. Takada 2014 2014, Phys. Rev. D 89, 083519 [arXiv/1401.0385]

D. Munshi, L. van Waerbeke, J. Smidt, P. Coles, 2012, MNRAS, 419, 536 |astro-ph/1103.1876|

D. Munshi, P. Valageas, L. Van Waerbeke, A. Heavens, 2008, Phys.Rept.462, 67 |astro-ph/0612667|

D. Munshi, J. Smidt, A. Heavens, P. Coles, A. Cooray, 2011, MNRAS, 411, 2241 lastro-ph/ $0001240 \mid$

D. Munshi, J. D. McEwen, T. Kitching, P. Fosalba, R. Teyssier, J. Stadel |arXiv/1902.04877|

D. Munshi, A. Heavens, P. Coles, 2011, MNRAS, 411, 2161 astro-ph/1002.2089]

D. Munshi, T. Kitching, A. Heavens, P. Coles, 2011, MNRAS, 416, 629 astro-ph/1012.3658]

D. Munshi, B. Jain, 2001, MNRAS, 322, 107 astro-ph/9912330

(c) 0000 RAS, MNRAS 000, 000-000 
D. Munshi, 2000, MNRAS, 318, 145 |astro-ph/0001240|

D. Munshi, A. Heavens, 2010, MNRAS, 401, 2406 [arxive/0904.4478]

D. Munshi, A. Heavens, A. Cooray, J. Smidt, P. Coles, P. Serra, 2011, MNRAS, 412, 1993 arxive/0910.3693

D. Munshi, B. Jain, 2000, MNRAS, 318, 109 astro-ph/9911502

D. Munshi, J. D. McEwen, T. Kitching, P. Fosalba, R. Teyssier, J. Stadel, [astro-ph/1902.04877]

D. Munshi, P. Valageas, A. J. Barber, 2004, MNRAS 350, 77 |astro-ph/0309698|

National Research Council. 2010. New Worlds, New Horizons in Astronomy and Astrophysics. The National Academies Press. https://doi.org/10.17226/12951.

M. Takada, B.Jain 2004, MNRAS, 348, 897 [astro-ph/0310125]

P. Valageas, D. Munshi, A. J. Barber, 2005, MNRAS. 356, 386 [astro-ph/0402227]

J. Byun, A. Eggemeier, D. Regan, D. Seery, R. E. Smith 2017, MNRAS, 471, 1581 |arXiv/1705.04392|

Y.-S. Song, A. Taruya, A. Oka JCAP 1508 (2015) 08, 007 |arXiv/1502.03099]

T. Namikawa, B. Bose, F. R. Bouchet, R. Takahashi, A. Taruya, |arxiv/1812.10635|

T. Namikawa, F. R. Bouchet, A. Taruya, |astro-ph/1805.10567]

Planck Collaboration, 2016, A\&A, 594, 17 [astro-ph/1502.01592]

Planck Collaboration, 2016, A\&A, 594A, 14, |arxive/1502.01590|

Planck Collaboration, 2018, [arXiv/1807.06205]

D. S. Salopek, J. R. Bond, 1990, PhRvD, 42, 3936

D. S. Salopek, J. R. Bond, 1991, PhRvD, 43, 1005

R. Scoccimarro, H. M. P. Couchman, 2001, MNRAS, 325, 4

R. Takahashi, T. Hamana, M. Shirasaki, T. Namikawa, T. Nishimichi, K. Osato, K. Shiroyama, 2017, ApJ, 850, 24 astro-ph/1706.01472]

J. A. Tyson, D. M. Wittman, J. F. Hennawi, D. N. Spergel, 2003, Nuclear Physics B Proceedings Supplements, 124, 21

P. Valageas, A. J. Barber, D. Munshi, 2004, MNRAS, 347, 654 [astro-ph/0303472]

A. J. Weiss, A. Schneider, R. Sgier, T. Kacprzak, A. Amara, A. Refregier, |arXiv/1905.1136|

\section{ACKNOWLEDGMENT}

DM is supported by a grant from the Leverhume Trust at MSSL. TDK is supported by Royal Society University Research Fellowship. RT is supported by MEXT/JSPS KAKENHI Grant No. JP15H05893 and 17H01131. BB acknowledges support from the Swiss National Science Foundation (SNSF) Professorship grant No. 170547.

\section{APPENDIX A: FITTING FUNCTIONS TO THE MATTER BISPECTRUM}

The fitting functions were provided by (Scoccimaro \& Couchman 2001, Gil-Marin et al. 2011). They have been tested in many different contexts. In a recent paper (Namikawa et al. 2018) they were used to model CMB lensing. Following earlier studies by various authors (Takada \& Jain 2004, Kilbinger \& Schneider 2005, Coulton et al. 2018), we use these functions to model weak lensing at low source redshifts. This is important given that the ongoing and future mission will be able to measure the lensing-induced bispectrum with high level of significance. This requires not only a (near) optimal data analysis pipeline but also an accurate theoretical modelling.

The perturbative matter bispectrum has the following form in terms of the perturbative kernels $F_{2}$ (Bernardeau et al 2002):

$$
B_{\delta}\left(\mathbf{k}_{1}, \mathbf{k}_{2}, \mathbf{k}_{3} ; \chi\right)=2 F_{2}\left(\mathbf{k}_{1}, \mathbf{k}_{2}, z\right) P_{\delta}\left(\mathbf{k}_{1}, z\right) P_{\delta}\left(\mathbf{k}_{2}, z\right)+\text { cyc.perm. }
$$

where $P_{\delta}(\mathbf{k}, z)$ is the matter power spectrum at a redshift $z$. The second order kernel $F_{2}$ can be computed using Eulerian perturbation theory which has the following form with $a=b=c=1$. However, comparison against numerical simulation shows that phenomenological extension is possible by making the paramaters $a, b$ and $c$ scale and redshift dependent (Scoccimaro \& Couchman 2001; Gil-Marin et al. 2011).

$$
F_{2}\left(\mathbf{k}_{1}, \mathbf{k}_{2}, z\right)=\frac{5}{7} a\left(k_{1}, z\right) a\left(k_{2}, z\right)+\frac{1}{2} \frac{k_{1}^{2}+k_{2}^{2}}{k_{1} k_{2}} b\left(k_{1}, z\right) b\left(k_{2}, z\right) \cos \theta+\frac{2}{7} c\left(k_{1}, z\right) c\left(k_{2}, z\right) .
$$


We list the functions that appear in the fitting function for completeness:

$$
\begin{aligned}
& a(k, z)=\frac{1+\sigma_{8}(z)^{a_{6}} \sqrt{0.7 Q\left(n_{\mathrm{eff}}\right)}\left(q a_{1}\right)^{n_{e f f}+a_{2}}}{1+\left(q a_{1}\right)^{n_{e f f}+a_{2}}} \\
& b(k, z)=\frac{1+0.2 a_{3}\left(n_{\mathrm{eff}}+3\right)\left(q a_{7}\right)^{n_{\mathrm{eff}}+3+a_{8}}}{1+\left(q a_{1}\right)^{n_{\mathrm{eff}}+3.5+a_{8}}} \\
& c(k, z)=\frac{1+\left[4.5 a_{4} /\left(1.5+\left(n_{\mathrm{eff}}+3\right)^{4}\right)\right]\left(q a_{5}\right)^{n_{\mathrm{eff}}+3+a_{9}}}{1+\left(q a_{5}\right)^{n_{\mathrm{eff}}+3.5+a_{9}}} .
\end{aligned}
$$

The amplitude of the bispectrum is fixed by the amplitude $Q(x)=\left(4-2^{x}\right) /\left(1+2^{x+1}\right)$ and $q=k / k_{\mathrm{NL}}$ with $k_{\mathrm{NL}}$ defined through the following equation $4 \pi k_{\mathrm{NL}}^{3} P^{\operatorname{lin}}\left(k_{\mathrm{NL}}\right)=1$ and $n_{\mathrm{eff}}=d \ln P^{\operatorname{lin}}(k) / d \ln k$. Two different fitting functions are available in the literature. An initial fitting function was suggested by Scoccimaro \& Couchman (2001). An improved fitting function was given by the following parameters in Gil-Marin et al. (2011) which we have used:

$a_{1}=0.484, a_{2}=3.74, a_{3}=-0.849, a_{4}=0.392, a_{5}=1.01, a_{6}=-0.575, a_{7}=0.128, a_{8}=-0.722, a_{9}=-0.926$.

It is expected that some modified gravity theories would also leave a detectable signature in convergence or $\kappa$ maps. However, the theoretical modelling requires an accurate framework. At present there are no fitting functions for modified gravity theories. Various heuristics schemes from perturbative calculations to phenomenological modification of Eq. (A1) have been developed recently (Bose \& Taruya 2018, Namikawa, Bouchet \& Taruya 2018).

\section{APPENDIX B: WEAK LENSING BISPECTRUM IN 3D}

With the advent of accurate photometric redshift information a full 3D analysis beyond tomographic binning has become possible. A 3D decomposition of the convergence maps can be accomplished using a spherical Fourier-Bessel transform following basis function. The forward and reverse transforms have the following form (Castro, Heavens \& Kitching 2005. Munshi et al. 2011):

$\kappa_{\ell m}(k)=\sqrt{\frac{2}{\pi}} \int d^{3} \mathbf{r} \kappa(\mathbf{r}) j_{\ell}(k r) Y_{\ell m}^{*}(\widehat{\boldsymbol{\Omega}}) ; \quad \kappa(\mathbf{r})=\sqrt{\frac{2}{\pi}} \int d k \sum_{\ell} \sum_{m=-\ell}^{\ell} \kappa_{\ell m}(k) j_{\ell}(k r) Y_{\ell m}(\widehat{\boldsymbol{\Omega}})$.

The 3D basis functions are constructed using the eigenfunctions of the Laplacian operator taking advantage of the radial symmetry $j_{\ell}$ above corresponds to the spherical Bessel function and $k$ corresponds to the radial wave number and $r=|\mathbf{r}|$ is the radial co-moving distance. The corresponding 2D bispectrum defined in Eq. 2a. -Eq. $2 \mathrm{~b}$ ) now gets generalised to:

$$
\begin{aligned}
& \left\langle\kappa_{\ell_{1} m_{1}}\left(k_{1}\right) \kappa_{\ell_{2} m_{2}}\left(k_{2}\right) \kappa_{\ell_{3} m_{3}}\left(k_{3}\right)\right\rangle=\left(\begin{array}{ccc}
\ell_{1} & \ell_{2} & \ell_{3} \\
m_{1} & m_{2} & m_{3}
\end{array}\right) \mathcal{B}_{\ell_{1} \ell_{2} \ell_{3}}\left(k_{1}, k_{2}, k_{3}\right) \\
& \mathcal{B}_{\ell_{1} \ell_{2} \ell_{3}}\left(k_{1}, k_{2}, k_{3}\right)=\sum_{m_{1} m_{2} m_{3}}\left(\begin{array}{ccc}
\ell_{1} & \ell_{2} & \ell_{3} \\
m_{1} & m_{2} & m_{3}
\end{array}\right)\left\langle\kappa_{\ell_{1} m_{1}}\left(k_{1}\right) \kappa_{\ell_{2} m_{2}}\left(k_{2}\right) \kappa_{\ell_{3} m_{3}}\left(k_{3}\right)\right\rangle .
\end{aligned}
$$

Following the same series of arguments the estimator presented in Eq.5. now gets generalised to:

$\mathcal{B}_{\ell_{1} \ell_{2} \ell_{3}}\left(k_{1}, k_{2}, k_{3}\right)=\left\langle\int d \Omega \mathcal{L}_{1}(\widehat{\boldsymbol{\Omega}}) \mathcal{L}_{2}(\widehat{\boldsymbol{\Omega}}) \mathcal{L}_{3}(\widehat{\boldsymbol{\Omega}})\right\rangle ; \quad \mathcal{L}_{i}(\widehat{\boldsymbol{\Omega}})=\sum_{m_{i}=-\ell_{i}}^{\ell_{i}} \kappa_{\ell_{i} m_{i}}\left(k_{i}\right) Y_{\ell_{i} m_{i}}(\widehat{\boldsymbol{\Omega}})$.

A binning in $k$ can be included to reduce the scatter as we have done for the multipoles $\ell$.

\section{APPENDIX C: BEYOND BISPECTRUM: SHAPES OF WEAK LENSING TRISPECTRUM}

The near all-sky weak lensing maps from surveys such as Euclid will also allow determination of non-Gaussianity statistics beyond the lowest-order, e.g., the fourth-order correlator or the trispectrum. The trispectrum can be useful not only to construct the covariance of the power spectrum estimator but also as a consistency check for the lower order estimators. In this section we will extend the estimator presented above for the bispectrum to the case of trispectrum.

(C) 0000 RAS, MNRAS 000, 000-000 


\section{Munshi et al.}

The trispectrum $T_{\ell_{3} \ell_{4}}^{\ell_{1} \ell_{2}}(L)$ is defined through the following expression $(\mathrm{Hu} 2001)$ :

$$
\begin{aligned}
& \left\langle\kappa_{\ell_{1} m_{1}} \kappa_{\ell_{2} m} \kappa_{\ell_{3} m_{3}} \kappa_{\ell_{4} m_{4}}\right\rangle=\sum_{m_{i}} \sum_{M}(-1)^{M}\left(\begin{array}{ccc}
\ell_{1} & \ell_{2} & L \\
m_{1} & m_{2} & M
\end{array}\right)\left(\begin{array}{ccc}
\ell_{3} & \ell_{4} & L \\
m_{3} & m_{4} & -M
\end{array}\right) T_{\ell_{3} \ell_{4}}^{\ell_{1} \ell_{2}}(L) ; \\
& T_{\ell_{3} \ell_{4}}^{\ell_{1} \ell_{2}}(L)=(2 L+1) \sum_{m_{i}} \sum_{M}\left(\begin{array}{ccc}
\ell_{1} & \ell_{2} & L \\
m_{1} & m_{2} & M
\end{array}\right)\left(\begin{array}{ccc}
\ell_{3} & \ell_{4} & L \\
m_{3} & m_{4} & -M
\end{array}\right)\left\langle\kappa_{\ell_{1} m_{1}} \kappa_{\ell_{2} m} \kappa_{\ell_{3} m_{3}} \kappa_{\ell_{4} m_{4}}\right\rangle .
\end{aligned}
$$

The Wigner 3j-symbols above ensure that the triangle inequalities imposed by statistical isotropy and homogeneity of the trispectrum in the harmonic space is represented by a quadrilateral. The indices (harmonics) $\ell_{1}, \ell_{2}, \ell_{3}, \ell_{4}$ represents the sides of the quadrilateral and the index $L$ represents one of the diagonal of the quadrilateral.

Using notations introduced before an estimator for the trispectrum can be expressed in the following form:

$$
\hat{T}_{\ell_{3} \ell_{4}}^{\ell_{1} \ell_{2}}(L)=\frac{2 L+1}{4 \pi} \frac{1}{h_{\ell_{1} \ell_{2} L}} \frac{1}{h_{\ell_{3} \ell_{4} L}} \sum_{p_{1}}^{N_{\text {pix }}} \sum_{p_{2}}^{N_{\text {pix }}} \mathcal{L}_{1}\left(\Omega_{p_{1}}\right) \mathcal{L}_{2}\left(\Omega_{p_{1}}\right) \mathcal{L}_{3}\left(\Omega_{p_{1}}\right) \mathcal{L}_{4}\left(\Omega_{p_{2}}\right) P_{L}\left(\Omega_{p_{1}} \cdot \Omega_{p_{2}}\right) .
$$

Here $P_{L}$ represents Legendre polynomial of order $L$ and $\widehat{\boldsymbol{\Omega}}_{p_{1}} \cdot \widehat{\boldsymbol{\Omega}}_{p_{2}}=\cos \theta_{12}$ where $\theta_{12}$ is the angle between the two unit vectors $\widehat{\boldsymbol{\Omega}}_{p_{1}}$ and $\widehat{\boldsymbol{\Omega}}_{p_{2}}$ representing the pixels $p_{1}$ and $p_{2}$. The presence of a double sum in Eq. C1c) makes it computationally demanding. However, estimates from degraded low resolution maps can be performed at a reasonable cost. The disconnected part of the trispectrum can be subtracted by using Gaussian maps that are generated using the same power spectrum even in the absence of noise. The contribution from (Gaussian) noise can be subtracted in an analogous manner.

Among various configurations that are typically pursued in the literature for the trispectrum are one-leg squeezed trispectrum, two-leg squeezed trispectrum and collapsed and flattened trispectrum. The positive trispectrum represents positive kurtosis and negative trispectra represent a negative kurtosis. Gravitational collapse generates a positive trispectrum.

Few comments are in order. The trapezium configuration represented by $\ell_{1}=\ell_{2}=\ell_{3}=\ell_{4} / 2$ and the kite configurations; represented by $2 \ell_{1}=2 \ell_{2}=\ell_{3}=\ell_{4}$ generalise the bispectrum configurations for a positive amplitude they correspond to elongated over-dense collapsed filament like structure surrounded by underdense regions. For the squeezed configurations the diagonally squeezed configurations correspond to $L<\ell_{1}, \ell_{2}, \ell_{3}, \ell_{4}$ and correspond to modulation of small scale power spectrum by large scale fluctuations. The one legged squeezed configuration represents large scale modulation of local or small-scale bispectrum and the two-legged squeezed configuration correspond to the case when bispectrum is zero and trispectrum is the leading order non-Gaussianity. In addition flattened configurations of trispectrum which corresponds to $\ell_{1}=\ell_{2}=\ell_{3}=\ell_{4} / 3$ provides signature that correspond to symmetric perturbations along a line in the real space very similar to a cosmic string. These configurations of the lensing induced CMB trispectrum has been studied in some detail (Lewis 2011). The corresponding investigation in the context of weak lensing at low redshift requires a numerical fitting function for the trispectrum beyond what can be achieved by perturbative approach. However, at present there is no suitable fitting formula to derive analytical expressions beyond the perturbative regime or its extensions using effective field theory (EFT) so we lack the corresponding formula for the weak lensing trispectrum. 